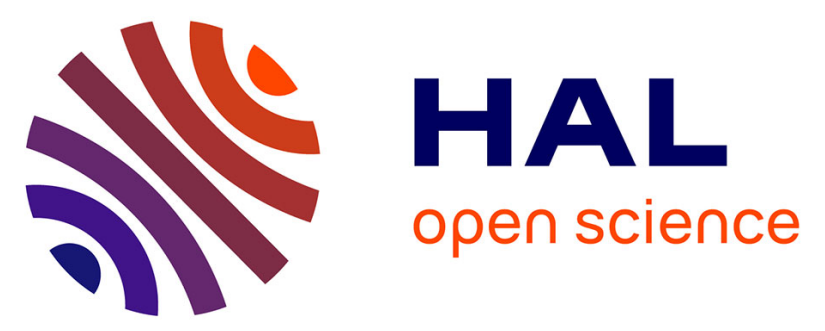

\title{
Impact of the Lund Integrated Medicines Management (LIMM) model on medication appropriateness and drug-related hospital revisits
}

Lina M. Hellström, Åsa Bondesson, Peter Höglund, Patrik Midlöv, Lydia Holmdahl, Eva Rickhag, Tommy Eriksson

\section{To cite this version:}

Lina M. Hellström, Åsa Bondesson, Peter Höglund, Patrik Midlöv, Lydia Holmdahl, et al.. Impact of the Lund Integrated Medicines Management (LIMM) model on medication appropriateness and drug-related hospital revisits. European Journal of Clinical Pharmacology, 2011, 67 (7), pp.741-752. 10.1007/s00228-010-0982-3 . hal-00669210

\section{HAL Id: hal-00669210 https://hal.science/hal-00669210}

Submitted on 12 Feb 2012

HAL is a multi-disciplinary open access archive for the deposit and dissemination of scientific research documents, whether they are published or not. The documents may come from teaching and research institutions in France or abroad, or from public or private research centers.
L'archive ouverte pluridisciplinaire HAL, est destinée au dépôt et à la diffusion de documents scientifiques de niveau recherche, publiés ou non, émanant des établissements d'enseignement et de recherche français ou étrangers, des laboratoires publics ou privés. 


\section{Impact of the Lund Integrated Medicines Management (LIMM) model on medication appropriateness and drug-related hospital revisits}

Lina Marie Hellström, Åsa Bondesson, Peter Höglund, Patrik Midlöv, Lydia Holmdahl, Eva Rickhag, Tommy Eriksson.

L. M. Hellström

eHealth Institute and School of Natural Sciences, Linnaeus University, Kalmar, Sweden

Å. Bondesson

Department of Clinical Pharmacology, Lund University, Lund, Sweden

Apoteket Farmaci AB, Hospital Pharmacy, Skåne University Hospital, Lund, Sweden

P. Höglund

Department of Clinical Pharmacology, Lund University, Lund, Sweden

P. Midlöv

Department of Clinical Sciences in Malmö, General practice/Family Medicine, Lund University, Lund, Sweden

Centre for Primary Health Care Research, Lund University, Lund, Sweden

L. Holmdahl

Department of Medicine, Skåne University Hospital, Lund, Sweden

E. Rickhag

Apoteket Farmaci AB, Hospital Pharmacy, Skåne University Hospital, Lund, Sweden

T. Eriksson

Department of Clinical Pharmacology, Lund University, Lund, Sweden 


\title{
Corresponding author
}

\author{
Lina Hellström
}

eHealth Institute

Linnaeus University

39182 Kalmar

SWEDEN

Phone: +46 480497175

Mobile phone: +46 733502106

e-mail: lina.hellstrom@1nu.se

The study was funded by Apoteket AB and the County of Skåne, Sweden.

\section{Abstract}

\section{Purpose}

To examine the impact of systematic medication reconciliations when admitted to hospital, and medication review while in hospital, on the number of inappropriate medications and unscheduled drug-related hospital revisits in elderly patients.

Methods

A prospective, controlled study in 210 patients, aged 65 years or older, who were admitted to one of three internal medicine wards at a University Hospital in Sweden. Patients received either standard care or care according to the Lund Integrated Medicines Management (LIMM) model. A multi-professional team, including a clinical pharmacist, provided medication reconciliations on admission and medication reviews during the hospital stay for the LIMM group. Blinded reviewers evaluated the appropriateness of the prescribing (using the Medication Appropriateness Index) on admission and discharge, and assessed the probability that a drug-related problem was the reason for any patient readmitted to hospital or visiting the emergency department within three months of discharge (using WHO causality criteria).

Results

There was a greater decrease in the number of inappropriate drugs in the intervention group than in the control group for both the intention-to-treat population (51\% [95\% CI 43-58\%] versus 39\% [95\% CI $30-48 \%$ ], $\mathrm{p}=0.0446)$ and the per-protocol population (60\% [95\% CI 51-67\%] versus 44\% [95\% CI 34- 
$52 \%$ ], $\mathrm{p}=0.0106)$. There were 6 revisits to hospital in the intervention group which were judged as 'possibly, probably or certainly drug-related', compared with 12 in the control group ( $\mathrm{p}=0.0469)$.

Conclusions

In this study, medication reconciliation and reviews provided by a clinical pharmacist in a multiprofessional team significantly reduced the number of inappropriate drugs and unscheduled drugrelated hospital revisits for elderly patients.

\section{Keywords}

Clinical pharmacy services

Medication Appropriateness Index

Medication review

Medication reconciliation

Drug-related problems

Inpatients 


\section{Introduction}

Drug treatment is an important component of medical care for the elderly and has the potential to decrease morbidity and mortality and to increase quality of life. However, problems associated with drug treatment are frequent among elderly hospitalised patients $[1,2]$ and, for some patients, it can be a drug-related problem that causes the hospital admission [3-5]. Many drug-related problems can be solved or prevented; it has been estimated that more than half of the drug-related admissions to hospital are preventable [6]. A number of different underlying causes of drug-related hospital admissions have been identified; for example, clinically inappropriate drug regimens, lack of sufficient monitoring and follow-up, insufficient quality of information transfer between levels of care, and poor adherence to drug treatment among patients. Given the complexity of the process leading to a drug-related hospital admission [6,7], it is likely that interventions for solving and preventing drug-related admissions and other negative consequences from drug therapy need to be multifaceted.

An integrated medicines management approach, involving input by clinical pharmacists at admission, during the hospital stay and at discharge, has been shown to be effective in reducing health care utilisation [8] and improving medication appropriateness $[9,10]$. We have developed the Lund Integrated Medicines Management (LIMM) model, which describes a systematic approach to individualising and optimising drug treatment in elderly inpatients. A more detailed description of the LIMM model is presented in Table 1. Medicines management is defined as a concept that seeks to maximise health through the optimum use of medical drugs [11] and the term 'integrated' indicates that different levels of care are involved, for example hospital and primary care. The model covers several aspects of the use of medications, from appropriate prescribing to the ways in which the drugs are taken or not taken by the patients [11]. Appropriate prescribing encompasses a range of values; three of the most important of these are the patient's wants, the pharmaceutical/pharmacological qualities of the drug and the wellbeing of the general population [12]. The Medication Appropriateness Index (MAI) is a validated instrument for measuring the pharmacological and economic aspects of prescribing appropriateness; the criteria used in the MAI can be described as a combination of 
implicit (judgment-based) and explicit (criterion-based) [13]. Results from an earlier study of the LIMM model showed positive effects on the appropriateness of drug therapy according to the MAI [10], but a larger controlled study was suggested to confirm the results and to study the effect on drug-related hospital admission levels.

The main objective of this study was to examine the impact of medication reconciliations when admitted to hospital and inpatient medication reviews, according to the LIMM model, on the number of inappropriate medications taken by elderly patients at discharge from hospital. The secondary objective was to examine the effect of LIMM-based patient care on the number of unscheduled drug-related hospital readmissions and emergency department visits within three months of hospital discharge.

\section{Methods}

\section{Patients and setting}

In this prospective controlled study, we compared patients receiving LIMM-based medication reconciliation and reviews (the intervention group) with patients receiving standard care (the control group).

The intervention was implemented in three internal medicine wards ( $\mathrm{A}, \mathrm{B}$ and $\mathrm{C})$ at Skåne University Hospital in Lund, Sweden, between January 2007 and March 2008 (Fig 1). The patients in all three wards received standard care until implementation of the LIMM intervention, which was started at different times in the respective wards (see Fig 1). All patients in wards A, B and C received the intervention after it had been implemented but the patients evaluated retrospectively for eligibility for inclusion in the study included only those who were staying in the study wards on November 1, 2006 (before the intervention), and March 1, 2007, November 1, 2007 and April 1, 2008 (about one month after implementation of the intervention in wards A, B and C, respectively). Patients were included if they: (1) were aged 65 years or older, and (2) had been prescribed at least one drug for regular use. Patients were excluded if they had been staying in the study wards during one of the previous 
inclusion dates. The regional Ethical Review Board did not consider an ethical approval to be necessary and had no objections to the study.

\section{Multi-professional team intervention according to the LIMM model}

During the intervention periods, clinical pharmacists conducted the medication reconciliation on admission of the patient to the ward and reviewed and monitored medication during the hospital stay according to the LIMM model. The admission medication reconciliation was performed on week-days, shortly after the patient had been admitted, using the LIMM Medication Interview Questionnaire parts 1-3 [14] (Table 1). Firstly, the most accurate patient medication list was identified (part 1 of the medication interview questionnaire). The current medication list in the electronic medical record was compared with lists from other sources, including the patients and their close relations, drug lists from primary or community care, and the national computerised pharmacy dispensing register. On the basis of identified discrepancies, the pharmacist suggested changes to the current drug list in a face-to-face discussion with a ward physician. Secondly, patients who took responsibility for their own drug treatment at home were interviewed about other aspects of their drug use in part 2 of the interview (Table 1). Finally, if the patient was capable and willing to participate, in-depth questions concerning adherence to the drug regimen [15] and beliefs about the medication [16] were added to the interview (part 3). Any problems concerning the patient's drug use emerging during the interview were addressed by the pharmacist by means of counselling and/or written information, including wardspecific drug information leaflets.

On week-days, before morning rounds, the pharmacists identified drug-related problems by conducting structured medication reviews, which addressed the following categories of risk: drugs that required therapeutic drug monitoring, less appropriate drug therapy, interchangeable drugs (according to a regional interchangeable list), problems with drug handling (e.g. swallowing, crushing, inhalation), drug interactions, type of drug or drug dose not adjusted according to liver/renal function, indication for drug treatment not known, natural remedy drugs, untreated indication and drugs causing adverse drug reactions. Information sources included the patient's medication list, medical record notes, laboratory values and 
other relevant data. During ward rounds, the drug-related problems which the pharmacist considered to be the most relevant were discussed within the multiprofessional team (physicians, nurses, carers and paramedics). Patients were followed up at least twice a week to enable identification of new drug-related problems and to monitor previously identified problems. All data were documented on the LIMM Medication Review Form (Table 1).

Clinical pharmacists did not work in the wards during the standard care control periods and no formal medication reviews were undertaken. In some cases, informal medication reconciliation at admission was undertaken but there were no tools or instructions for this.

During both control and intervention periods, patients and primary or community care personnel received a Discharge Information Form which included a Medication Report describing changes in drug treatment during the hospital stay and a Medication List (Table 1). This discharge medication reconciliation was not part of the evaluation because control patients received identical information at discharge and the benefit of this procedure has already been demonstrated $[17,18]$.

\section{Data collection and outcome measures}

The primary outcome measure was the change in the number of drugs with at least one inappropriate score $(\mathrm{C})$ between admission and discharge, according to the MAI (see below). Secondary outcome measures included the number of patients revisiting the hospital because of a drug-related complaint within three months of discharge and MAI scores for each patient and drug at discharge.

\section{Medication appropriateness index (MAl)}

The 10 items that comprise the MAI are indication, drug effectiveness, correct dosage, correct directions, practical directions, drug-drug interactions, drug-disease interactions, drug duplication, duration of treatment and expense. Each drug was rated as appropriate (A), marginally appropriate (B) or inappropriate (C) for each of the 10 items [13]. In the analysis, ratings for individual items were dichotomised as appropriate (A or B) versus inappropriate (C). A drug was categorised as 
inappropriate if it received a $\mathrm{C}$ for at least one of the ten MAI items. According to a weighting scheme, a summed MAI score of between 0 and 18 can be produced for each medication [19] and a patient MAI score can be calculated by summing the scores of all medications for each patient. The literature indicates that the overall inter-rater reliability of this index is moderate to very good and the overall intra-rater reliability is good to very good $[13,19-22]$. The content validity has also been shown to be good [18]. In some cases, however, the reliability of a few specific individual MAI items has been fair to poor [20]. Clinical judgment is needed to assess some items, although the rating process has been standardised by including explicit instructions on how to interpret each of the 10 items [13].

The MAI assessments were done retrospectively by a research assistant (junior clinical pharmacist, MSc Pharm) who was not involved in the care of the patients and who had been trained in the use of the MAI by an experienced clinical pharmacist and researcher (Figure 2). The MAI was applied to drugs taken regularly; topical drugs, laxatives and drugs taken as needed were excluded. Patient data used in the MAI assessment of drugs at admission included the drug list at admission, laboratory and diagnostic test results, and medical record notes from admission and the first day in hospital. The research assistant was blinded with respect to group allocation.

The MAI assessments for drugs at discharge were performed in two steps for the intervention group (Figure 2) and one step for the control group. In step 1, the control and intervention patients were assessed using the drug list at discharge, the medical record notes during the hospital stay, the laboratory and diagnostic test results and the discharge summary. The research assistant performing the assessment was blinded with respect to group allocation. In step 2, the intervention patients were reassessed; patient information documented in the pharmacist's Medication Review Form was made available to the reviewer and the MAI scores for drugs receiving an inappropriate rating in step 1 were re-evaluated based on this information. As medication review forms were only produced for intervention patients, the research assistant could not remain blinded to group allocation for this step. The step 2 assessment was performed because some information in the Medication Review Forms had not been documented in the patient's medical record by the physicians, which could potentially have changed the MAI scores. Only the results from the first 
step of the discharge MAI assessment (the blinded evaluation) were used in the main analysis, as we wanted to avoid bias.

\section{Drug-related hospital revisits}

Data collected from the electronic medical records of patients who had an unscheduled readmission or visit to the emergency department at the Skåne University Hospital in Lund within three months of discharge were used as a basis for evaluation of the reason for the revisit to hospital. If a patient had several unscheduled revisits to hospital within three months, only data from the first contact were used.

In the reviewing process, we combined clinical judgment with the use of predetermined triggers, namely combinations of drugs and symptoms or certain "highalert" medications [3-5,23,24]. As shown in Figure 2, the initial review, which was performed by a research assistant supported by an experienced clinical pharmacist, was followed by a final review by a multi-professional expert group of senior researchers and clinicians (one clinical pharmacist, one clinical pharmacologist, one consultant in internal medicine/geriatrics and one general practitioner). The initial reviewers were as inclusive as possible when choosing cases for further review; cases were classified as 'potentially drug-related' and reviewed by the expert group if there was even the smallest suspicion of a drug-related revisit.

The expert group classified the type of drug-related problem as adverse drug reaction, therapeutic failure (including non-compliance, dose reduction/discontinuation, dose too low, drug interaction, inadequate therapeutic drug monitoring) or untreated indication. The cases were further classified by using the WHO criteria for causality [25]; the causal relationship between the drug treatment and a symptom or diagnosis present at the hospital revisit was classified as 'certain', 'probable', 'possible' or 'unlikely'. In addition, the contribution of the drug-related problem to the hospital revisit was assessed and classified as 'dominant', 'partly contributing', 'less important' or 'not contributing' [26]. Drug-related problems associated with medications that had been newly initiated in the outpatient setting after discharge from hospital were excluded from the assessment, as we only wanted to evaluate the treatment that was a result of hospital care during the study. All reviewers in the initial and final reviews were blinded with respect to group allocation. In the 
statistical analysis, the hospital revisits were classified as drug-related only when 'possible, 'probable' or 'certain' drug-related cases were also classified as the 'dominant cause' for or 'partly contributing' to the revisit.

\section{Statistics}

To assess the comparability of study groups at study inclusion, we used Student's ttest, the Mann-Whitney test and Fisher's exact test when appropriate. The difference in the number of drugs with inappropriate ratings was analysed by Poisson regression, with an offset equal to the natural logarithm of the number of drugs with an inappropriate rating at admission. Because the natural logarithm for zero does not exist, all zeros in the offset variable were set to 0.3. Our choice was based on comparisons of a number of values between 0.01 and 0.5 , which showed that 0.3 gave the best fit of the model to the data. We also judged it to be an appropriate distance from 1. The Mann-Whitney test was used when comparing MAI scores between the intervention and control groups. For the MAI calculations, bootstrap 95\% confidence intervals for the means were obtained by using the re-sampling technique [27]. The main analyses of the MAI results were performed according to the intention-to-treat principle, using the 'baseline observation carried forward' for missing observations. A per-protocol analysis of patients who had received the complete intervention (both medication reconciliation and review) and who did not die in hospital was also carried out. The Chi squared test for trend was used to analyse drug-related revisits to hospital. The significance level in all analyses was set to 0.05 . The $\mathrm{R}$ language and environment for statistical computing program (www.r-project.org) was used for the statistical analysis.

The power calculation performed for the primary objective was based on the results of a previous study of the LIMM model in 53 patients, which showed that the number of drugs with at least one inappropriate rating decreased by $33 \%$ in the intervention group and $7 \%$ in the control group [10]. We believed that it would be valuable to detect an even smaller difference between the groups and wanted to account for loss to follow-up; after performing a number of simulations, we therefore decided to include at least 100 patients in each group. 


\section{Results}

Of the 232 patients eligible for inclusion, 210 were enrolled in the study (Figure 3). Fifteen patients in the intervention group did not receive the complete intervention, due to short length of stay in hospital, no clinical pharmacist working at weekends, or closed wards due to infection outbreak among the patients. In addition, twelve intervention patients (including two who did not receive the complete intervention) and nine control patients died during the initial hospital stay. Eighty-four intervention patients and 92 control patients were therefore included in the per-protocol analysis. Demographic data at admission to hospital (baseline) are shown in Table 2. Gender, age and number of drugs at admission were similar in both groups. The median (interquartile range) length of stay in hospital was 16 (9-28) days in the intervention group and 13 (8-21) days in the control group ( $\mathrm{p}=0.0905)$. A current medication list was identified for all 97 patients who received medication reconciliation (part 1 of the LIMM Medication Interview Questionnaire). In addition, 23 of these 97 patients were interviewed about knowledge of their medication and adherence to the drug regimen (part 2 of the questionnaire) and 17 of these 23 also completed the questionnaire concerning adherence to the drug regimen [15] and the other on beliefs about medicines [16] (part 3).

\section{Medication Appropriateness Index}

\section{Drugs with at least one inappropriate rating}

There was a greater decrease in the number of drugs with at least one inappropriate rating in the intervention group than in the control group (51\% [95\% CI 43-58\%] versus 39\% [95\% CI 30-48\%], p=0.0446), according to the intention-to-treat analysis of the MAI assessment, step 1. At discharge, $18.4 \%$ of the drugs for intervention patients and $21.9 \%$ of the drugs for controls were rated as inappropriate.

In the per-protocol analysis, the number of drugs with at least one inappropriate rating decreased by $60 \%$ (95\% CI 51-67\%) in the intervention group, compared with $44 \%$ (95\% CI 34-52\%) in the control group ( $\mathrm{p}=0.0106)$. At discharge, $15.1 \%$ of the drugs for the intervention group and $20.2 \%$ of the drugs for the control group were rated as 
inappropriate. The mean number of drugs with at least one inappropriate rating per patient, at admission and discharge, is shown in Table 3.

The mean number of inappropriate drugs changed between admission and discharge as a result of discontinuing inappropriate drugs, initiating inappropriate drugs, adjusting existing therapy to more appropriate therapy (for example, by changing the dose), or improving documentation (for example, stating the indication for a specific drug in the medical record). The number of inappropriate drugs that were discontinued, initiated or adjusted is shown in Table 4.

In the MAI assessment, step 2 (performed in the intervention group at discharge), 117 drugs for 62 patients (all drugs with at least one $\mathrm{C}$ rating, according to the MAI assessment, step 1) were assessed. The MAI rating was changed from a $\mathrm{C}$ to an $\mathrm{A}$ or B for 27 drugs in 19 patients. The average decrease per patient between step 1 and 2 in number of drugs with at least one $\mathrm{C}$ was 0.44 (95\% CI 0.26-0.63).

\section{MAl items}

Table 5 shows that the MAI item "expense" received the most inappropriate ratings in both control and intervention patients, followed by "indication" and "duration". When the indication was rated as inappropriate, both expense and duration also received an inappropriate rating, according to the MAI instructions. In the intervention group, there were improvements in all MAI criteria except "effectiveness" and "correct directions" (Table 5). In the control group, there was an improvement for six of the ten criteria.

\section{MAl scores}

The average MAI scores per patient and per drug, at admission and discharge, are shown in Table 3. No significant differences between the control and intervention groups at discharge were found in the patient MAI score (intention-to-treat $\mathrm{p}=0.553$; per-protocol $\mathrm{p}=0.421$ ), or the MAI score per drug (intention-to-treat $\mathrm{p}=0.543$; perprotocol $\mathrm{p}=0.329$ ). 


\section{Drug-related revisits to the hospital}

Within three months of discharge, 71 patients were readmitted (unscheduled admissions) to hospital and 15 visited the emergency department. Two patients (one intervention and one control) were excluded from the analysis, due to insufficient medical record data. There were 6 revisits to hospital with a causality assessment of possible, probable or certain in the intervention group compared with 12 in the control group ( $\mathrm{p}=0.0469)$ (Table 6). Among the drug-related cases, 3 patients visited the emergency department without being admitted to hospital and 15 patients were admitted to a hospital ward.

In $5.6 \%$ of intervention patients (6 of 108) and $12.0 \%$ of control patients (12 of 100), the revisit to hospital was at least possibly drug related ( $\mathrm{p}=0.138$, Fisher's exact test). The absolute risk reduction for possibly drug-related revisits was $6.4 \%$ (95\% CI: $1.2 \%$ to $14.1 \%$ ). The cases where a drug-related problem was assessed as having a certain or probable relationship with the revisit to hospital are described in more detail in Table 7.

\section{Discussion}

The results of this study showed that medication reviews and medication reconciliation on admission to hospital, carried out according to the LIMM model, were associated with a significant decrease in the number of inappropriate drugs taken by elderly hospitalised patients. In agreement with these results, a previous study showed a reduction in the number of drugs with at least one inappropriate rating after implementation of the LIMM model [10]. A number of earlier controlled studies on pharmaceutical care, integrated medicines management or other collaborative approaches in hospitalised patients have also shown improvements in the appropriateness of medications (decrease in the MAI scores) [9,28,29].

In accordance with the previous study of the LIMM model [10], we regarded 'the number of drugs with at least one inappropriate rating', to be the most suitable main outcome measure. This measure has also previously been used by others [20,28]. By using that measure we could avoid summing or subtracting scores, which is generally 
not recommended when dealing with ordered categorical data (i.e. the MAI scores A, B and C) [30]. Nonetheless, we have presented the MAI scores in this study in order to facilitate comparison with previous studies. In our study, the average patient MAI score in the intervention group at discharge was lower than that in most other studies (indicating more appropriate prescribing) $[9,10,28,29]$. However, because the control group also had a very low patient MAI score, there were no apparent differences between the groups. In two previous interventions with a similar lack of improvement in the MAI scores [29,31] neither the intervention nor the control group improved.

In our main analysis we used the most conservative parameters possible, i.e. MAI scores from the blinded assessment (step 1), results from the intention-to-treat population, and "baseline observation carried forward" for missing data. Despite this, the results still indicated a difference between the groups. When the per-protocol population and the nonblinded MAI assessment were analysed, the difference between the groups was larger, suggesting that the effect of the intervention may have been underestimated in the main analysis. The use of a blinded outcome assessment, as in our study and two other hospital-based MAI studies [29,31], strengthens the validity of the MAI ratings in comparison with those from other studies, which used reviewers who were not blind to group allocation $[9,10,28]$.

The clinical consequences of a reduction in the number of inappropriate drugs are difficult to estimate, since the evidence for an association between inappropriate prescribing and adverse patient outcomes is mixed and contradictory [32]. However, the two published studies on the predictive validity of the MAI both indicate that this tool can reliably predict clinical patient outcomes [33,34]. Lund et al. showed that inappropriate prescribing, according to a modified MAI score [33], is associated with adverse drug events in older men, and Schmader et al. demonstrated an association between worse MAI scores and poorer blood pressure control and use of more health services [34].

Our study has some potential limitations. Firstly, the nonrandomised design could have introduced selection bias. Secondly, the study was undertaken solely in wards of internal medicine, limiting the generalisability of the results. With this in mind and to improve the generalisability, we developed a structured process for performing 
medication review and reconciliation, and used structured forms (i.e. checklists and questionnaires). Finally, instead of performing two or more independent outcome assessments for each patient, a single rater or a single group of raters assessed the patient data. We decided to use only one rater for the MAI assessments, as previous studies of MAI have shown a good or very good agreement within raters [13,19-22] and the main purpose of this study was to quantify changes in MAI (where intra-rater agreement was more important [21]). The group of reviewers evaluating the drugrelated revisits to hospital was chosen to provide varied backgrounds and expert knowledge in order to minimize the risk of misclassifying the revisits. In addition, they used checklists for detecting drug-related problems and two different explicit criteria to classify the cases.

In a number of cases, the comprehensiveness and quality of the documentation in the medical records affected the appropriateness of the medication. Adding information from the medication review forms led to a change in the MAI rating from 'inappropriate' to 'appropriate' for $23 \%$ of the drugs at discharge. We have already showed that communicating changes in the medication list, together with the reasons for these changes, to the patient and the next level of care decreases medication errors and the number of health care contacts $[17,18]$. We suggest that there is potential to improve the quality of prescribing even more if a broader range of issues concerning medication appropriateness and drug-related problems is documented and communicated to the next level of care in a structured way.

We found a significant reduction in the number of unscheduled drug-related revisits within three months of discharge from hospital. Similarly, a recent randomized controlled study of a comparable intervention, also in Sweden, showed a reduction in all revisits to hospital as well as in drug-related readmissions [35], and patients who received an integrated medicines management service in Northern Ireland benefited from a decreased rate of readmission and an increased time to readmission [8]. It is probable that the effect of the entire LIMM intervention (at admission, during the hospital stay and at discharge) on reduced need for medical care due to drug-related problems will approximate the sum of the effects of the discharge medication reconciliation (Discharge Information including a Medication Report and a 
Medication List) previously shown [17] and the admission medication reconciliation and medication reviews as demonstrated in this study.

Our findings confirm the effect on medication appropriateness previously shown by Bergkvist et al. in a study of the entire LIMM model [10]. In contrast to that study, however, our study demonstrated a relatively large improvement in the number of inappropriate drugs from admission to discharge in both the control and intervention groups. The large improvement seen in the control group could be the result of an effect of the discharge medication reconciliation process on medication appropriateness, since this activity was implemented in both groups.

In conclusion, the results of this study indicate that medication reconciliation and reviews, according to the integrated medicines management model demonstrated here, can reduce the number of inappropriate drugs taken by elderly patients during a hospital stay and the number of subsequent unscheduled drug-related hospital visits.

\section{Acknowledgement}

We thank the pharmacists Tanja Lehtinen and Lily Esporlas for skilful help with the collection and evaluation of data. We would also like to thank the staff of the study wards and the clinical pharmacists at the Hospital pharmacy, Skåne University Hospital in Lund, Sweden.

\section{Conflict of interest}

The authors declare that they have no conflicts of interest.

\section{References}

1. Blix HS, Viktil KK, Reikvam A, Moger TA, Hjemaas BJ, Pretsch P, Vraalsen TF, Walseth EK (2004) The majority of hospitalised patients have drug-related problems: results from a prospective study in general hospitals. Eur J Clin Pharmacol 60 (9):651-658. doi:10.1007/s00228-004-0830-4 2. Krahenbuhl-Melcher A, Schlienger R, Lampert M, Haschke M, Drewe J, Krahenbuhl S (2007) Drugrelated problems in hospitals: a review of the recent literature. Drug Saf 30 (5):379-407. doi:3053 [pii] 
3. van der Hooft CS, Dieleman JP, Siemes C, Aarnoudse AJ, Verhamme KM, Stricker BH, Sturkenboom MC (2008) Adverse drug reaction-related hospitalisations: a population-based cohort study. Pharmacoepidemiol Drug Saf 17 (4):365-371. doi:10.1002/pds.1565

4. Pirmohamed M, James S, Meakin S, Green C, Scott AK, Walley TJ, Farrar K, Park BK, Breckenridge AM (2004) Adverse drug reactions as cause of admission to hospital: prospective analysis of 18820 patients. BMJ 329 (7456):15-19. doi:10.1136/bmj.329.7456.15 329/7456/15 [pii]

5. Mjorndal T, Boman MD, Hagg S, Backstrom M, Wiholm BE, Wahlin A, Dahlqvist R (2002) Adverse drug reactions as a cause for admissions to a department of internal medicine. Pharmacoepidemiol Drug Saf 11 (1):65-72. doi:10.1002/pds.667 6. Howard RL, Avery AJ, Howard PD, Partridge M (2003) Investigation into the reasons for preventable drug related admissions to a medical admissions unit: observational study. Qual Saf Health Care 12 (4):280-285

7. Howard R, Avery A, Bissell P (2008) Causes of preventable drug-related hospital admissions: a qualitative study. Qual Saf Health Care 17 (2):109-116. doi:17/2/109 [pii]

10.1136/qshc. 2007.022681

8. Scullin C, Scott MG, Hogg A, McElnay JC (2007) An innovative approach to integrated medicines management. J Eval Clin Pract 13 (5):781-788. doi:JEP753 [pii]

10.1111/j.1365-2753.2006.00753.x

9. Burnett KM, Scott MG, Fleming GF, Clark CM, McElnay JC (2009) Effects of an integrated medicines management program on medication appropriateness in hospitalized patients. Am J Health Syst Pharm 66 (9):854-859. doi:66/9/854 [pii]

10.2146/ajhp080176

10. Bergkvist A, Midlov P, Hoglund P, Larsson L, Eriksson T (2009) A multi-intervention approach on drug therapy can lead to a more appropriate drug use in the elderly. LIMM-Landskrona Integrated Medicines Management. J Eval Clin Pract 15 (4):660-667. doi:JEP1080 [pii] 10.1111/j.1365-2753.2008.01080.x

11. Simpson D (2001) What is medicines management and what is pharmaceutical care? . Pharm J 266 (7133):150

12. Cribb A, Barber N (1997) Prescribers, patients and policy: the limits of technique. Health Care Anal 5 (4):292-298. doi:10.1007/BF02678527

13. Hanlon JT, Schmader KE, Samsa GP, Weinberger M, Uttech KM, Lewis IK, Cohen HJ, Feussner JR (1992) A method for assessing drug therapy appropriateness. J Clin Epidemiol 45 (10):1045-1051. doi:0895-4356(92)90144-C [pii]

14. Bondesson A, Hellstrom L, Eriksson T, Hoglund P (2009) A structured questionnaire to assess patient compliance and beliefs about medicines taking into account the ordered categorical structure of data. J Eval Clin Pract 15 (4):713-723. doi:JEP1088 [pii] 10.1111/j.1365-2753.2008.01088.x

15. Morisky DE, Green LW, Levine DM (1986) Concurrent and predictive validity of a self-reported measure of medication adherence. Med Care 24 (1):67-74 
16. Horne R, Weinman J, Hankins M (1999) The beliefs about medicines questionnaire: The development and evaluation of a new method for assessing the cognitive representation of medication. Psychol Health 14:1-24

17. Midlov P, Deierborg E, Holmdahl L, Hoglund P, Eriksson T (2008) Clinical outcomes from the use of Medication Report when elderly patients are discharged from hospital. Pharm World Sci 30 (6):840845. doi:10.1007/s11096-008-9236-1

18. Bergkvist A, Midlov P, Hoglund P, Larsson L, Bondesson A, Eriksson T (2009) Improved quality in the hospital discharge summary reduces medication errors--LIMM: Landskrona Integrated Medicines Management. Eur J Clin Pharmacol 65 (10):1037-1046. doi:10.1007/s00228-009-0680-1 19. Samsa GP, Hanlon JT, Schmader KE, Weinberger M, Clipp EC, Uttech KM, Lewis IK, Landsman PB, Cohen HJ (1994) A summated score for the medication appropriateness index: development and assessment of clinimetric properties including content validity. J Clin Epidemiol 47 (8):891-896. doi:0895-4356(94)90192-9 [pii]

20. Spinewine A, Dumont C, Mallet L, Swine C (2006) Medication appropriateness index: reliability and recommendations for future use. J Am Geriatr Soc 54 (4):720-722. doi:JGS668_8 [pii] 10.1111/j.1532-5415.2006.00668_8.x

21. Stuijt CC, Franssen EJ, Egberts AC, Hudson SA (2009) Reliability of the medication appropriateness index in Dutch residential home. Pharm World Sci 31 (3):380-386. doi:10.1007/s11096-009-9283-2

22. Kassam R, Martin LG, Farris KB (2003) Reliability of a modified medication appropriateness index in community pharmacies. Ann Pharmacother 37 (1):40-46

23. The National Board of Health and Welfare (2003) Indikatorer för utvärdering av kvaliteten i äldres läkemedelsterapi- Socialstyrelsens förslag. Publication no. 2003-110-20. http://www.socialstyrelsen.se/Publicerat/2004/1578. Accessed Feb 2009

24. Institute for safe medication practices (2008) List of high-alert medications. Available at: http://www.ismp.org/Tools/highalertmedications.pdf Accessed Feb 2009

25. The use of the WHO-UMC system for standardised case causality assessment. http://www.whoumc.org/graphics/4409.pdf. Accessed Feb 2009

26. Hallas J, Harvald B, Gram LF, Grodum E, Brosen K, Haghfelt T, Damsbo N (1990) Drug related hospital admissions: the role of definitions and intensity of data collection, and the possibility of prevention. J Intern Med 228 (2):83-90

27. Efron B, Tibshirani R (1993) An Introduction to the Bootstrap. Chapman \& Hall, New York 28. Spinewine A, Swine C, Dhillon S, Lambert P, Nachega JB, Wilmotte L, Tulkens PM (2007) Effect of a collaborative approach on the quality of prescribing for geriatric inpatients: a randomized, controlled trial. J Am Geriatr Soc 55 (5):658-665. doi:JGS1132 [pii]

10.1111/j.1532-5415.2007.01132.x

29. Schmader KE, Hanlon JT, Pieper CF, Sloane R, Ruby CM, Twersky J, Francis SD, Branch LG, Lindblad CI, Artz M, Weinberger M, Feussner JR, Cohen HJ (2004) Effects of geriatric evaluation and 
management on adverse drug reactions and suboptimal prescribing in the frail elderly. Am J Med 116 (6):394-401. doi:10.1016/j.amjmed.2003.10.031

S0002934303007526 [pii]

30. Altman DG (1991) Practical statistics for medical research. Chapman and Hall, London

31. Bucci C, Jackevicius C, McFarlane K, Liu P (2003) Pharmacist's contribution in a heart function clinic: patient perception and medication appropriateness. Can J Cardiol 19 (4):391-396

32. Spinewine A, Schmader KE, Barber N, Hughes C, Lapane KL, Swine C, Hanlon JT (2007)

Appropriate prescribing in elderly people: how well can it be measured and optimised? Lancet 370 (9582):173-184. doi:S0140-6736(07)61091-5 [pii]

10.1016/S0140-6736(07)61091-5

33. Lund BC, Carnahan RM, Egge JA, Chrischilles EA, Kaboli PJ (2010) Inappropriate prescribing predicts adverse drug events in older adults. Ann Pharmacother 44 (6):957-963. doi:aph.1M657 [pii] 10.1345/aph.1M657

34. Schmader KE, Hanlon JT, Landsman PB, Samsa GP, Lewis IK, Weinberger M (1997) Inappropriate prescribing and health outcomes in elderly veteran outpatients. Ann Pharmacother 31 (5):529-533

35. Gillespie U, Alassaad A, Henrohn D, Garmo H, Hammarlund-Udenaes M, Toss H, Kettis-Lindblad A, Melhus H, Morlin C (2009) A comprehensive pharmacist intervention to reduce morbidity in patients 80 years or older: a randomized controlled trial. Arch Intern Med 169 (9):894-900.

doi:169/9/894 [pii]

10.1001/archinternmed.2009.71

\section{Figure legends}

Fig. 1 The Lund Integrated Medicines Management (LIMM) model was implemented at different times on the three wards during the year 2007 to 2008. A sample of patients was collected retrospectively and assessed for eligibility. Dates for inclusion of study patients were chosen before and about one month after implementation of the intervention. We included 23 control patients and 55 intervention patients from ward A, 28 control and 36 intervention patients from ward B and 50 control and 18 intervention patients from ward $\mathrm{C}$.

Fig. 2 Interventions during the hospital stay and evaluations made after hospital discharge in the intervention and control groups, respectively. MAI, Medication Appropriateness Index; DRP, DrugRelated Problem.

Fig. 3 Flow of patients through the study 
${ }^{a}$ Some patients did not receive the full intervention because of short length of stay in hospital, death during hospital stay, no clinical pharmacist working at weekends, or closed wards due to infection outbreak among the patients.

${ }^{b}$ Patients lost to follow-up died during the hospital stay. The total of 12 patients in the intervention group who died in hospital includes two who had also not received the complete intervention.

${ }^{c}$ One patient from each group was excluded from the analysis, due to insufficient medical record data. 
Table 1 The Lund Integrated Medicines Management (LIMM) model. The table summarises the activities performed in the hospital wards for each patient, the responsible professional groups, and the tools used to perform the activities

\begin{tabular}{|c|c|c|c|c|}
\hline When & How often & Activity & Responsibility & Tool \\
\hline $\begin{array}{l}\text { At } \\
\text { admission }\end{array}$ & $\begin{array}{l}\text { Once for each } \\
\text { patient }\end{array}$ & $\begin{array}{l}\text { Admission medication } \\
\text { reconciliation }\end{array}$ & $\begin{array}{l}\text { Clinical } \\
\text { pharmacist }\end{array}$ & $\begin{array}{l}\text { LIMM Medication Interview Questionnaire, parts 1-3 [14], according to } \\
\text { medication, disease and patient characteristics: } \\
\text { Part 1: identification of the most accurate medication list for the patient } \\
\text { Part 2: addition of questions concerning the patient's practical handling and } \\
\text { knowledge of the medications and adherence to the medical regimen } \\
\text { Part 3: addition of more detailed questions concerning the patient's adherence to } \\
\text { the medical regimen [15] and beliefs about the medications [16] }\end{array}$ \\
\hline \multirow{3}{*}{$\begin{array}{l}\text { During } \\
\text { hospital } \\
\text { stay }\end{array}$} & \multirow{3}{*}{$\begin{array}{l}\text { At regular } \\
\text { intervals for } \\
\text { each patient }\end{array}$} & $\begin{array}{l}\text { Medication review and } \\
\text { monitoring }\end{array}$ & $\begin{array}{l}\text { Clinical } \\
\text { pharmacist }\end{array}$ & LIMM Medication Review Form \\
\hline & & Symptom assessment & $\begin{array}{l}\text { Nurse (or clinical } \\
\text { pharmacist) }\end{array}$ & LIMM Symptom Assessment Form \\
\hline & & $\begin{array}{l}\text { Lead the team and organise a } \\
\text { treatment plan based on the } \\
\text { symptom assessment, } \\
\text { medication review and } \\
\text { reconciliation results }\end{array}$ & Physician & Documented in the patient health record \\
\hline \multirow[t]{2}{*}{$\begin{array}{l}\text { At } \\
\text { discharge }\end{array}$} & $\begin{array}{l}\text { Once for each } \\
\text { patient }\end{array}$ & $\begin{array}{l}\text { Discharge medication } \\
\text { reconciliation }\end{array}$ & Physician & $\begin{array}{l}\text { LIMM Discharge Information Form, including a Medication Report and a } \\
\text { Medication List (a list of current drugs, doses and indications) }\end{array}$ \\
\hline & Occasionally $^{\mathrm{a}}$ & $\begin{array}{l}\text { Quality control of discharge } \\
\text { medication reconciliation }\end{array}$ & $\begin{array}{l}\text { Clinical } \\
\text { pharmacist }\end{array}$ & LIMM Quality Control form for Discharge Medication Reconciliation \\
\hline
\end{tabular}

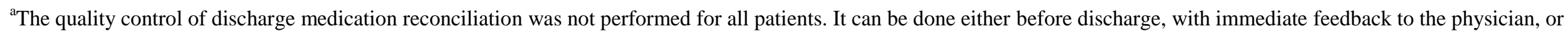
retrospectively. 
Table 2 Characteristics of patients in the intervention and control groups at admission (baseline)

\begin{tabular}{lll}
\hline Characteristic & $\begin{array}{l}\text { Intervention group } \\
(\mathrm{n}=109)\end{array}$ & $\begin{array}{l}\text { Control group } \\
(\mathrm{n}=101)\end{array}$ \\
\hline $\begin{array}{l}\text { Gender [no. (\%)] } \\
\quad \text { Female }\end{array}$ & $60(55.0)$ & $51(50.5)$ \\
$\quad 49(45.0)$ & $50(49.5)$ \\
$\quad$ Male & $83.0(7.0)$ & $81.8(7.4)$ \\
Age in years [mean (SD)] & $8(5-11)$ & $7(5-11)$ \\
$\begin{array}{l}\text { No. of drugs in continuous use } \\
\text { [median (IQR)] }\end{array}$ & $1(1-3)$ & $1(1-3)$ \\
$\begin{array}{l}\text { No. of drugs for use on demand } \\
\text { [median (IQR)] }\end{array}$ & & \\
\hline
\end{tabular}

$\mathrm{IQR}=$ interquartile range; $\mathrm{SD}=$ standard deviation. 
Table 3 Number of drugs with at least one inappropriate rating per patient according to the Medication Appropriateness Index (MAI), MAI score per patient and MAI score per drug at admission and discharge. Results from both the intention-to-treat (ITT) analysis (n=210) and the per-protocol (PP) analysis (n=176) are presented. Higher MAI scores indicate a higher level of inappropriateness. Figures are means (95\% confidence intervals)

\begin{tabular}{|c|c|c|c|c|c|}
\hline & \multicolumn{3}{|c|}{ Intervention group $($ ITT $n=109 ;$ PP $n=84)$} & \multicolumn{2}{|c|}{ Control group (ITT $n=101 ;$ PP $n=92)$} \\
\hline & Admission & & arge & Admission & Discharge \\
\hline & & Step $1^{\mathrm{a}}$ & Step 2 & & \\
\hline ITT & $3.0(2.5-3.5)$ & $1.5(1.2-1.8)$ & $1.2(0.9-1.6)$ & $2.8(2.3-3.3)$ & $1.7(1.3-2.1)$ \\
\hline $\mathrm{PP}$ & $2.9(2.4-3.4)$ & $1.2(0.9-1.4)$ & $0.8(0.6-1.1)$ & $2.7(2.3-3.2)$ & $1.6(1.2-1.9)$ \\
\hline \multicolumn{6}{|l|}{ Patient MAI score ${ }^{\mathrm{b}}$} \\
\hline ITT & $12.5(10.1-15.0)$ & $4.5(3.2-6.2)$ & $3.5(2.3-5.1)$ & $10.8(8.9-13.1)$ & $4.9(3.6-6.4)$ \\
\hline PP & $12.2(9.7-14.9)$ & $3.2(2.3-4.2)$ & $1.9(1.4-2.6)$ & $10.7(8.7-13.0)$ & $4.3(3.1-5.5)$ \\
\hline \multicolumn{6}{|l|}{ MAI score per drug ${ }^{\mathrm{c}}$} \\
\hline ITT & $1.5(1.2-1.8)$ & $0.5(0.4-0.6)$ & $0.4(0.3-0.5)$ & $1.3(1.1-1.5)$ & $0.5(0.4-0.6)$ \\
\hline PP & $1.5(1.2-1.9)$ & $0.4(0.3-0.5)$ & $0.2(0.2-0.3)$ & $1.3(1.1-1.5)$ & $0.5(0.3-0.6)$ \\
\hline
\end{tabular}

${ }^{\mathrm{a}}$ In the analysis of the primary outcome (number of inappropriate drugs), the discharge MAI assessment step 1 data for the intervention group were compared with data for the control group.

${ }^{\mathrm{b}}$ The patient MAI score is the sum of the scores for all drugs for each patient. 
${ }^{\mathrm{c}}$ The MAI score per drug is the sum of the scores for all drugs divided by the number of drugs evaluated. 
Table 4 Number of inappropriate drugs at admission and discharge and number (percent) of inappropriate drugs that were discontinued, adjusted or initiated during the hospital stay (intentionto-treat analysis)

\begin{tabular}{|c|c|c|c|}
\hline \multirow[b]{3}{*}{$\begin{array}{l}\text { Number of inappropriate drugs } \\
\text { at admission }\end{array}$} & \multicolumn{2}{|c|}{$\begin{array}{c}\text { Intervention group } \\
\text { (Admission: } \mathrm{n}=858 \mathrm{drugs} \\
\text { Discharge: } \mathrm{n}=868 \text { ) }\end{array}$} & \multirow[t]{2}{*}{$\begin{array}{c}\text { Control group } \\
\text { (Admission: } n=783 \text { drugs } \\
\text { Discharge: } n=785 \text { ) }\end{array}$} \\
\hline & Step 1 & Step 2 & \\
\hline & 324 & 324 & 279 \\
\hline Discontinued $^{\mathrm{a}}$ & $69(21)$ & $69(21)$ & $48(17)$ \\
\hline $\begin{array}{l}\text { Therapy adjusted or } \\
\text { documentation } \\
\text { improved }^{\mathrm{a}}\end{array}$ & $123(38)$ & $144(44)$ & $87(31)$ \\
\hline $\begin{array}{l}\text { Number of inappropriate drugs } \\
\text { at discharge }\end{array}$ & 160 & 133 & 172 \\
\hline Initiated in hospital ${ }^{\mathrm{b}}$ & $28(18)$ & $22(17)$ & $28(16)$ \\
\hline
\end{tabular}

${ }^{\mathrm{a}}$ Number (percent of the number of inappropriate drugs at admission)

${ }^{\mathrm{b}}$ Number (percent of the number of inappropriate drugs at discharge) 
Table 5 Percentage of drugs that were inappropriate for each MAI criterion at admission and discharge, according to the intention-to-treat analysis

\begin{tabular}{|c|c|c|c|c|c|}
\hline \multirow[t]{3}{*}{ MAI criteria } & \multicolumn{3}{|c|}{ Intervention group, 109 patients } & \multicolumn{2}{|c|}{ Control group, 101 patients } \\
\hline & \multirow{2}{*}{$\begin{array}{l}\text { Admission } \\
(\mathrm{n}=858 \\
\text { drugs }) \\
\end{array}$} & \multicolumn{2}{|c|}{$\begin{array}{l}\text { Discharge } \\
\text { (n=868 drugs) }\end{array}$} & \multirow{2}{*}{$\begin{array}{l}\text { Admission } \\
(\mathrm{n}=783 \\
\text { drugs })\end{array}$} & \multirow{2}{*}{$\begin{array}{l}\text { Discharge } \\
(\mathrm{n}=785 \\
\text { drugs }) \\
\end{array}$} \\
\hline & & Step 1 & Step 2 & & \\
\hline Expense & 26.5 & 8.9 & 6.9 & 24.3 & 10.6 \\
\hline Indication & 24.1 & 6.5 & 4.6 & 20.6 & 6.5 \\
\hline Duration & 24.0 & 6.5 & 4.6 & 20.6 & 6.5 \\
\hline Drug-disease interaction & 5.8 & 4.0 & 4.0 & 5.9 & 5.9 \\
\hline Dosage & 5.0 & 1.5 & 0.8 & 4.6 & 2.4 \\
\hline Drug-drug interaction & 3.5 & 2.1 & 1.4 & 2.7 & 1.7 \\
\hline Practical directions & 2.2 & 2.2 & 2.1 & 1.5 & 1.9 \\
\hline Duplication & 1.0 & 0.8 & 0.7 & 1.5 & 1.8 \\
\hline Effectiveness & 0.7 & 0.8 & 0.8 & 0.8 & 0.9 \\
\hline Correct directions & 0.7 & 0.7 & 0.5 & 0.8 & 0.4 \\
\hline Overall $^{\mathrm{a}}$ & 37.8 & 18.4 & 15.3 & 35.6 & 21.9 \\
\hline
\end{tabular}

${ }^{\mathrm{a}}$ Inappropriate rating in at least 1 of the 10 criteria. 
Table 6 Assessment of causality (using WHO criteria [25]) and contribution (using Hallas' criteria [26]) of drug-related problems to the hospital visit for all revisits to hospital. The WHO criteria for causality comprised: certain, probable, possible or unlikely. Hallas' criteria comprised: dominant, partly contributing, less important and not contributing. Types of drug-related problems covered were: adverse drug reaction, therapeutic failure (including non-compliance, dose-

reduction/discontinuation, too low dose, drug interaction, inadequate therapeutic drug monitoring) and untreated indication. Figures given indicate number of patients

Classification according to causality and contribution Intervention group Control group $(\mathrm{n}=40)$ $(n=44)$

Certain and dominant

0

1

Probable and dominant or partly contributing

2

5

Possible and dominant or partly contributing

4

6

Unlikely or less important/not contributing

38

28 
Table 7 Details of patient cases where a drug-related problem was assessed as having a certain or probable relationship with the revisit to hospital

\begin{tabular}{|c|c|c|c|c|c|c|c|}
\hline Allocation & $\begin{array}{l}\text { Symptoms/diagnoses at } \\
\text { admission }\end{array}$ & Drugs involved & Causality & Contribution to revisit & $\begin{array}{l}\text { Type of drug-related } \\
\text { problem }\end{array}$ & Type of revisit & $\begin{array}{l}\text { Time to } \\
\text { revisit (days) }\end{array}$ \\
\hline \multirow[t]{3}{*}{$\mathrm{C}$} & Diminished peripheral & Metoprolol & Probable & Partly contributing & Adverse drug reaction & Readmission & 4 \\
\hline & circulation, foot pain & Tramadol & Possible & Partly contributing & & & \\
\hline & Sedation and fall & Zolpidem & Possible & Partly contributing & & & \\
\hline $\mathrm{C}$ & $\begin{array}{l}\text { Hypoglycaemia, fatigue, } \\
\text { sweating, confusion }\end{array}$ & $\begin{array}{l}\text { Insulin lispro and } \\
\text { insulin glargine }\end{array}$ & Certain & Dominant & $\begin{array}{l}\text { Adverse drug reaction } \\
\text { and inadequate TDM }\end{array}$ & ED visit & 13 \\
\hline $\mathrm{C}$ & Tachycardia, atrial fibrillation & Digoxin & Probable & Dominant & $\begin{array}{l}\text { Therapeutic failure } \\
\text { (discontinuation) }\end{array}$ & ED visit & 20 \\
\hline $\mathrm{C}$ & $\begin{array}{l}\text { Hyperglycaemia, thirst, } \\
\text { dizziness }\end{array}$ & Insulin aspart & Probable & Dominant & $\begin{array}{l}\text { Therapeutic failure (low } \\
\text { adherence) }\end{array}$ & Readmission & 21 \\
\hline $\mathrm{C}$ & $\begin{array}{l}\text { Diminished peripheral } \\
\text { circulation and pale, cold feet }\end{array}$ & Atenolol & Probable & Partly contributing & Adverse drug reaction & Readmission & 68 \\
\hline $\mathrm{C}$ & $\begin{array}{l}\text { Respiratory distress, } \\
\text { deterioration of heart failure }\end{array}$ & Furosemide & Probable & Dominant & $\begin{array}{l}\text { Therapeutic failure } \\
\text { (discontinuation) }\end{array}$ & ED visit & 92 \\
\hline I & $\begin{array}{l}\text { Orthostatic hypotension, fall, } \\
\text { fracture }\end{array}$ & Metoprolol & Probable & Partly contributing & Adverse drug reaction & Readmission & 4 \\
\hline I & Hyperkalaemia & $\begin{array}{l}\text { Spironolactone } \\
\text { combined with } \\
\text { candesartan }\end{array}$ & Probable & Dominant & Adverse drug reaction & Readmission & 31 \\
\hline
\end{tabular}

C, control; I, intervention; ED, Emergency department; TDM, Therapeutic drug monitoring 